\title{
The Impact of Elevation Angle on MIMO Capacity
}

\author{
Mansoor Shafi*, Min Zhang ${ }^{\dagger}$, Peter J. Smith ${ }^{\dagger}$, Aris L. Moustakas ${ }^{\ddagger}$ and Andreas F. Molisch ${ }^{\S}$ \\ *Telecom New Zealand Ltd, 49-55 Tory Street, Wellington, New Zealand \\ Email: mansoor.shafi@telecom.co.nz \\ ${ }^{\dagger}$ Department of Electrical and Computer Engineering \\ University of Canterbury, Private Bag 4800, Christchurch, New Zealand \\ Email: $\{$ mzh22,p.smith\}@elec.canterbury.ac.nz \\ $\ddagger$ Department of Physics, University of Athens, Greece \\ Email: arislm@phys.uoa.gr \\ $\S$ Department of Electroscience, Lund University, Box 118, SE-221 00 Lund, Sweden \\ Email: andreas.molisch@ieee.org
}

\begin{abstract}
Many channel models for MIMO systems have appeared in the literature. However, with the exception of a few recent results, they are largely focussed on two dimensional (2D) propagation, i.e., propagation in the horizontal plane, and the impact of elevation angle is not considered. The assumption of $2 \mathrm{D}$ propagation breaks down when in some propagation environments the elevation angle distribution is significant. Consequently, the estimation of ergodic capacity assuming a 2D channel coefficient alone can lead to erroneous results. In this paper, for cross polarized channels, we define a composite channel model and channel coefficient that takes into account both 2D and 3D propagation. Using this composite channel coefficient we assess the ergodic channel capacity and discuss its sensitivity to a variety of different azimuth and elevation power distributions and other system parameters.
\end{abstract}

Index Terms-MIMO, cross polarized channels, angular power distribution, polarization, capacity.

\section{INTRODUCTION}

Cross-polarized MIMO systems have the potential to double the numbers of antenna while keeping the space requirements unchanged compared to co-polarized antennas. However, only few studies are available on how the propagation channels impact the capacity of such systems. In particular, the combined impact of the polarization characteristics and the elevation spectrum has been given little attention. In a recent paper [1], we introduced a composite, dual-polarized channel model that represents the total propagation channel as the sum of a $2 \mathrm{D}$ component (corresponding to radiation that is scattered far away from the transceiver), and a 3D component (corresponding to radiation scattered in the vicinity of the transceiver). This paper also analyzed the impact of this model on the capacity for a particular distribution of the $2 \mathrm{D}$ and the $3 \mathrm{D}$ components.
There are only few measurements of the angular distribution of the 2D and 3D components of the dualpolarized MIMO propagation channel available. The classic papers of [2], [3] have shed some light on polarization diversity. However, both these papers are for single input multiple output (SIMO) systems and are primarily aimed at assessing the benefits of polarization diversity in different environments. Also, they do not consider the impact of the elevation spectrum.

In a recent study, the $3 \mathrm{GPP}$ and $3 \mathrm{GPP} 2$ developed a cross-polarized 2D channel model for MIMO systems [4]. In another recent study [5], the elevation spectrum and cross-polarization discrimination (XPD) were measured in different radio propagation environments in Finland. The measured elevation spectrum was modeled by an asymmetrical double exponential distribution that had different parameters for the elevation and azimuth domains. However, this study only considered 3D propagation. In an earlier study, using a somewhat different 3D channel model, in urban Tokyo [6], a Gaussian elevation spectrum is proposed and measurements are used to derive an expression for the mean effective gain of a mobile antenna. In [7], a family of functions is proposed for the elevation spectrum but there is no measurement data. In general there is a real scarcity of published models for the elevation spectrum, channel models and parameters for propagation which includes a 3D component. Hence, it is highly important to investigate the sensitivity of key MIMO parameters, especially capacity, to the specifics of the propagation environment.

In [1], we have shown that ergodic capacity is sensitive to the power of the 3D component. However, this conclusion was obtained for the case where the AoA azimuth distribution in the 2D component follows a von Mises distribution and the AoA elevation distribution in the 3D 
component follows a spherically uniform distribution. No elevation consideration was made for the $2 \mathrm{D}$ component. The azimuth distribution of the $3 \mathrm{D}$ component was assumed to be uniform. This study is referred to as the base line case in this paper. In this paper, we wish to test the robustness of the base line study for a number of other azimuth and elevation distributions. Specifically, we address the following questions:

- Given a spherically uniform AoA distribution of the elevation in the $3 \mathrm{D}$ component, how sensitive is the ergodic capacity to the choice of AoA azimuth distributions of the 2D component? We simulate four cases: uniform, Laplacian, Gaussian and standard von Mises and also consider two different angle spreads (AS values) for the last three distributions.

- With a 2D AoA azimuth distribution fixed as standard von Mises, how sensitive is ergodic capacity to the choice of elevation distributions in the 3D component. We simulate four cases: elevation angle is spherical uniform, power is concentrated close to the horizontal plane of the scattering sphere, elevation angle is uniform and power concentrated in the top and bottom of the scattering sphere.

- Finally, for the above cases, we investigate the sensitivity of ergodic capacity to variation in a number of other parameters, i.e AS for the different AoA distributions and the relative power in the 2D/3D components.

Results show, that when keeping the 3D AoA distribution as spherical uniform, the ergodic capacity is very sensitive to the relative proportions of the $2 \mathrm{D}$ and $3 \mathrm{D}$ components, especially when the AoA azimuth distribution has low AS. This conclusion is valid for all the distributions considered. Furthermore, when fixing the $2 \mathrm{D}$ AoA azimuth distribution but changing the 3D elevation distributions, the ergodic capacity is higher than the base line when the power concentrates in the top and bottom of the scattering sphere.

The paper is organized as follows. Section II describes the new generalized composite channel model that takes into account the modeling of the 3D component. The simulation cases considered are given in Section III and the results are contained in Section IV. Finally, some conclusions are given in Section V.

\section{Generalized Model For Cross-Polarized CHANNELS}

We base our studies on the 3GPP spatial channel model (SCM) [4]. In this section, we will briefly describe this model and generalize it to take into account the effects of $3 \mathrm{D}$ propagation and polarization mixing. The

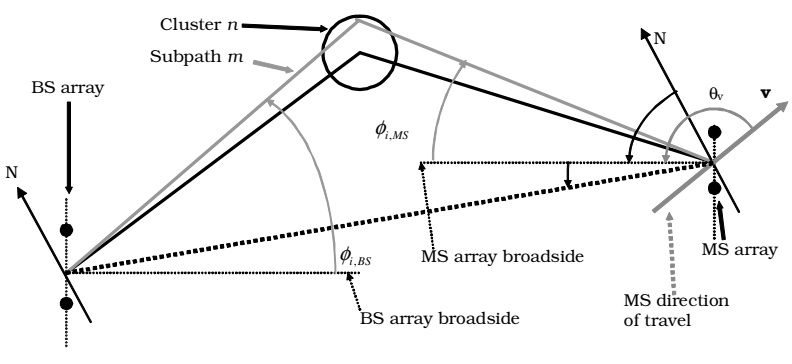

Fig. 1. Simplified SCM model for 2D case

total propagation channel is modeled as the sum of a 2D and a 3D component. This model is motivated by physical considerations in macro- and microcellular scenarios. A part of the radiation interacts only with objects that are far away from both the BS and the MS (e.g., far-away buildings, mountains, etc.); this radiation is incident mainly in the horizontal plane at both the BS and the MS. Another part of the radiation interacts with objects close to the MS (e.g., walls and furniture in a room, or a car chassis in which the MS is located); this part shows a much wider range of elevation angles.

\section{A. Modeling the $2 D$ component}

The 2D model is based on the SCM model. The SCM considers $\mathrm{N}$ time-resolvable paths, each corresponding to a cluster and each with $\mathrm{M}$ unresolvable subpaths. However for simplicity, we focus here on a single path. A simplified sketch of the model is given in Fig. 1.

For the modeling of cross polarized channels, one needs to model four channels between BS and MS antennas, namely those connecting the horizontal/vertical polarization at the to the horizontal/vertical polarization at the MS. Throughout the paper, we assume ideal tilted dipole antennas at both ends of the link. We also assume all the polarization mixing is due to the path effects i.e., we neglect antenna polarization leakage propagation effects. Thus, for example, an ideal dipole antenna tilted at angle $\alpha$ from the $z$-axis (see Fig. 2), has vertical and horizontal components of the antenna pattern which are proportional to

$$
\chi(\mathbf{k})=\left[\begin{array}{c}
\chi^{v}(\mathbf{k}) \\
\chi^{h}(\mathbf{k})
\end{array}\right] e^{i \mathbf{k r}}=\left[\begin{array}{c}
\cos \alpha \\
\sin \alpha \cos \phi
\end{array}\right] e^{i \mathbf{k r}}
$$

where, for compactness, we have included the overall phase factor for the incoming wave in the response of the antenna. $\mathbf{r}$ is the vector signifying the position of the antenna with respect to the center of the antenna array. The actual position of the center of the array is not important, since only relative distances between antennas play a role in the model. The vector $\mathbf{k}=2 \pi / \lambda[\cos \phi, \sin \phi, 0]$ is the $3 \mathrm{D}$ wave-vector of the direction of the incoming 
(or outgoing) wave, with the carrier wavelength $\lambda$ and $\phi$ is the azimuth angle of incoming/outgoing waves.

For simplicity, we focus on a single path. The fading channel coefficient $h_{s u}^{2 D}(t)$ of the path between BS antenna $s$ and MS antenna $u$ as given in [4] is then given by

$$
\begin{aligned}
h_{s u}^{2 D}(t)=\sqrt{\frac{1}{M}} \sum_{i=1}^{M}( & \chi_{s, B S}^{\dagger}\left(\mathbf{k}_{i, B S}\right) \mathbf{H}_{i}^{2 D} \\
& \left.\times \chi_{u, M S}\left(\mathbf{k}_{i, M S}\right) e^{-i \mathbf{k}_{i, M S} \cdot \mathbf{v} t}\right)
\end{aligned}
$$

with the superscript $2 D$ indicating wave propagation in 2 dimensions. In the above, $\chi$ are the antenna response vectors for the BS and MS antennas as defined in (1) and the azimuth angles $\phi$ in (1) are given by $\phi_{i, B S}$ and $\phi_{i, M S}$ for the BS and MS respectively, as in Fig 1. It should be stressed that this equation is valid for arbitrary antenna patterns, not necessarily ideal dipole antennas. Note that the square norm of $\chi, G(\mathbf{k})=|\chi(\mathbf{k})|^{2}$, is the antenna gain in the direction k. Also, $\mathbf{k}_{i, M S}$ and $\mathbf{k}_{i, B S}$ in (2) are the random plane wavevectors of the incoming and outgoing waves for each wave component $i=1, \ldots, M$, both assumed to be in the horizontal plane. $\mathbf{v}$ is the velocity vector of the MS and $t$ is time. In addition, $\mathbf{H}_{i}^{2 D}$ is a $2 \times 2$ matrix containing the random coefficients of the $i=1, \ldots, M$ wave components given by

$$
\mathbf{H}_{i}^{2 D}=\left[\begin{array}{cc}
z_{i}^{v v} & \sqrt{r_{1}} z_{i}^{v h} \\
\sqrt{r_{2}} z_{i}^{h v} & z_{i}^{h h}
\end{array}\right] .
$$

The $z_{i}$ terms in (3) are the random coefficients of the $i$ th wave component for each of the four polarization channels HH, HV, VH, VV. In the SCM model [4] and in this paper, these terms are defined as iid complex exponentials instead of complex Gaussians, for computational efficiency. $r_{1}$ and $r_{2}$ are inverse XPD values for $\mathrm{VV} / \mathrm{VH}$ and $\mathrm{HH} / \mathrm{HV}$ respectively, i.e, $r_{1}$ is the power of the $\mathrm{VH}$ component relative to the $\mathrm{VV}$ component. Values of $r_{1}$ and $r_{2}$ are set according to the SCM model [4].

\section{B. Modeling the $3 D$ component}

3D components are particularly important when analyzing cross-correlations between antennas with very different 3D response patterns. In these cases, focusing only on the radiation arriving in the horizontal plane (azimuth) may produce erroneous results. Therefore, we need to modify the above 2D model to incorporate such effects. From the random orientation of the MS antenna in the azimuth, it is reasonable to assume the azimuth distribution in 3D is uniform. In this paper, we consider a number of elevation angle distributions. The 3D model below enables us to model these situations.

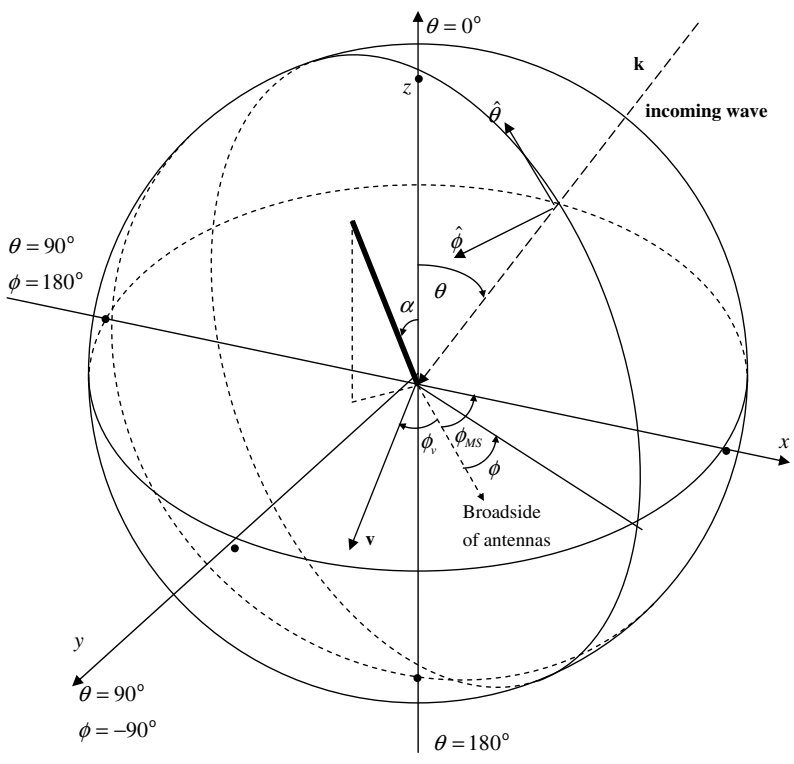

Fig. 2. Spherical coordinate system for 3D model

The 3D model requires a spherical coordinate system as shown in Fig. 2. In order to take into account the full three-dimensional antenna response of the MS antennas, we express the response vector $\chi$ in its $\theta$ and $\phi$ components as:

$$
\begin{aligned}
\chi_{M S}(\mathbf{k}) & =\left[\begin{array}{l}
\chi^{\theta}(\mathbf{k}) \\
\chi^{\phi}(\mathbf{k})
\end{array}\right] \\
& =\left[\begin{array}{c}
\cos \alpha \sin \theta+\sin \alpha \cos \theta \sin \phi \\
\sin \alpha \cos \phi
\end{array}\right] e^{i \mathbf{k r}}
\end{aligned}
$$

where $\chi^{\theta}, \chi^{\phi}$ are the $\theta$ and $\phi$ polarized responses of the antenna at direction $\mathbf{k}$. The vector $\mathbf{k}$ is defined in terms of $\theta$ and $\phi$ as [6], [8]:

$$
\mathbf{k}=2 \pi / \lambda[\sin \theta \cos \phi, \sin \theta \sin \phi, \cos \theta]
$$

as shown in Fig. 2. Note that in the limit of horizontal wave propagation, i.e., when $\theta=\pi / 2$, we recover the form of antenna response shown in (1).

The strength of the 3D radiation at the MS relative to that of the already existing $2 \mathrm{D}$ radiation depends on several factors. For example, in indoor scenarios it may include the distance of the MS from openings (e.g. windows), antenna heights of the BS etc. Since the surfaces from which these waves emanate towards the MS are of no particular geometry, we expect the horizontal and vertical components of the radiation to have been fully mixed. Hence we assume $X P D=1$ as in the uniform spherical case [9]. We assume this is also the case for the other cases of elevation spectrum simulated as we do not have any published sources of data on the XPD-elevation relationship. We may write 
the 3D fading channel coefficient $h_{s u}^{3 D}(t)$ for propagation between BS antenna $s$ and MS antenna $u$ as:

$$
\begin{array}{r}
h_{s, u}^{3 D}(t)=\sqrt{\frac{1}{M} \sum_{i=1}^{M}\left(\chi_{s, B S}^{\dagger}\left(\mathbf{k}_{i, B S}\right) \mathbf{H}_{i}^{3 D}\right.} \\
\left.\chi_{u, M S}\left(\mathbf{k}_{i, M S}\right) e^{-i \mathbf{k}_{i, M S} \cdot \mathbf{v} t}\right)
\end{array}
$$

with the superscript $3 D$ indicating wave propagation in 3 dimensions. The $\mathbf{k}_{i, M S}$ are independently chosen for the $2 \mathrm{D}$ and $3 \mathrm{D}$ channel coefficients and the matrix $\mathbf{H}_{i}^{3 D}$ for the $i$ th wave component is given by

$$
\mathbf{H}_{i}^{3 D}=\left[\begin{array}{cc}
z_{i}^{v \theta} & z_{i}^{v \phi} \\
z_{i}^{h \theta} & z_{i}^{h \phi}
\end{array}\right]
$$

The $z_{i}$ terms in (7) are the random coefficients of the $i$ th wave component for each of the $\mathrm{V}$ and $\mathrm{H}$ channels and their respective components in the $\theta$ and $\phi$ polarizations respectively. The antenna responses, $\chi$, for the BS are the same for both 2D and 3D models. At the MS, the antenna responses are different due to the $3 \mathrm{D}$ character of the radiation. As in the case of (2), equation (6) is valid for arbitrary antenna patterns. Nevertheless, for concreteness we focus on the simple case of ideal dipole antennas.

\section{Modeling the composite channel}

The composite channel coefficient between antennas $s$ and $u$ is modeled as the folowing scaled mixture of 2D and 3D channel coefficients:

$$
h_{s u}(t)=\sqrt{\frac{1}{1+g}} h_{s u}^{2 D}(t)+\sqrt{\frac{g}{1+g}} h_{s u}^{3 D}(t)
$$

where $g$ is the ratio of powers of the 3D to 2D components. There are very few measurements of $g$. For the case of indoor mobiles, which are far from open spaces, e.g. windows, one can assume that $g=\infty$, i.e., one can keep only the 3D components of the channel. For indoor channels close to a window, a reasonable value is $g=-4 d B$ [10]. We wish to study how the ergodic capacity varies with the value of $g$ for the different AoA 2D and 3D distributions in azimuth and elevation.

\section{Simulation Cases Considered}

Table I describes the simulations undertaken. In the left part of Table I, the 2D AoA distributions are changed whilst the 3D AoA distribution is held as uniform in azimuth and spherically uniform in elevation. In the right part, the 2D AoA distribution is held as standard von Mises in azimuth and the 3D AoA distributions are varied in elevation. In all cases the 3D AoA azimuth distribution is kept uniform.

The 2D AoA distributions are shown in Fig. 3. Note that the uniform density over $(-\pi, \pi]$ gives an AS of

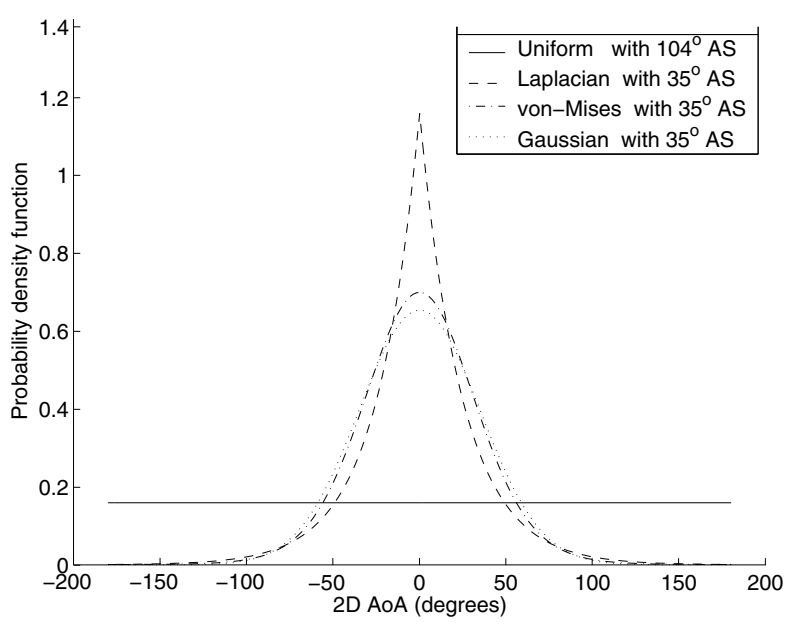

Fig. 3. Azimuth AoA distributions for the $2 \mathrm{D}$ component

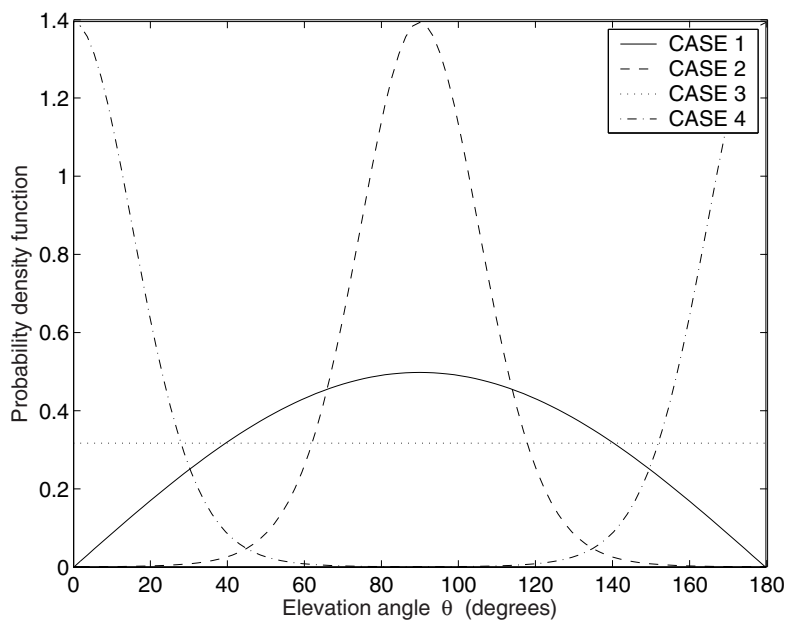

Fig. 4. Elevation AoA distributions for the 3D component

$104^{\circ}$. The 3D elevation AoA distributions are shown in Fig. 4 In Fig. 3, the von Mises, Laplacian and Gaussian distributions all have the same AS and are therefore expected to perform similarly when considering the impact on the ergodic capacity. In Fig. 4, case 1 is the base line, i.e., the elevation angle is spherically uniform. Case 2 has more incoming power in the horizontal plane. Case 3 is when all the incoming rays are uniformly distributed. Case 4 is when the incoming power is concentrated around the top and bottom of the scattering sphere. In our simulations, we have assumed the horizontal and vertical components to have been fully mixed and therefore the corresponding XPD to be equal to unity [1]. We note that in practice, XPD is not constant and depends on a number of parameters, e.g. distance, delay spread, the elevation and azimuth angles etc., see [1] and references therein. However, at present we have no simple way of deriving this composite dependence. 


\begin{tabular}{|c|c|c|c|c|c|c|c|c|c|c|}
\hline \multicolumn{5}{|c|}{ Parameters for AoA distributions: varying 2D component } & \multicolumn{6}{|c|}{ Parameters for AoA distributions: varying 3D component } \\
\hline \multicolumn{2}{|c|}{ 2D Component } & \multicolumn{3}{|c|}{ 3D component, $\phi \in(-p i, \pi], \theta \in(0, \pi]$} & \multicolumn{3}{|c|}{ 2D Component } & \multicolumn{3}{|c|}{ 3D component, $\phi \in(-p i, \pi], \theta \in(0, \pi]$} \\
\hline Dist. & AS & Dist. & $\phi$ pdf & $\theta$ pdf & CASE & Dist. & AS & Dist. & $\phi$ pdf & $\theta$ pdf \\
\hline $\mathrm{U}$ & $104^{\circ}$ & SU & $\frac{1}{2 \pi}$ & $\frac{\sin \theta}{2}$ & 1 & VM & $35^{\circ}$ & SU & $\frac{1}{2 \pi}$ & $\frac{\sin \theta}{2}$ \\
\hline Lap & $35^{\circ}, 2^{\circ}$ & SU & $\frac{1}{2 \pi}$ & $\frac{\sin \theta}{2}$ & 2 & VM & $35^{\circ}$ & SNU & $\frac{1}{2 \pi}$ & $\frac{\exp (\kappa \cos 2(\theta-\pi / 2))}{\pi I_{0}(\kappa)}, \kappa=3.365$ \\
\hline Gau & $35^{\circ}, 2^{\circ}$ & SU & $\frac{1}{2 \pi}$ & $\frac{\sin \theta}{2}$ & 3 & VM & $35^{\circ}$ & SNU & $\frac{1}{2 \pi}$ & $\frac{1}{\pi}$ \\
\hline VM & $35^{\circ}, 2^{\circ}$ & SU & $\frac{1}{2 \pi}$ & $\frac{\sin \theta}{2}$ & 4 & VM & $35^{\circ}$ & SNU & $\frac{1}{2 \pi}$ & $\frac{\exp (\kappa \cos 2 \theta)}{\pi I_{0}(\kappa)}, \kappa=3.365$ \\
\hline
\end{tabular}

TABLE I

PARAMETERS FOR AOA DISTRIBUTIONS

SU:SPHERICAL UNIFORM, SNU:SPHERICAL NON-UNIFORM, U:UNIFORM, LAP:LAPLACIAN, GAU: GAUSSIAN, VM:VON-MISES

\section{RESULTS AND DISCUSSIONS}

We now use the AoA distributions in Table I to calculate ergodic capacity and then use this calculation to determine the impact of the various azimuth and elevation distributions on the ergodic capacity. The ergodic capacity is given by the classic equation [11]

$$
\mathcal{C}=E\left[\log _{2} \operatorname{det}\left(\mathbf{I}_{n_{M S}}+\frac{S N R}{n_{B S}} \mathbf{H H}^{\dagger}\right)\right]
$$

where expectation is over realizations of the channel matrix $\mathbf{H}$ and the BS is the transmitter. It is assumed that channel state information is only known at the receiver. The ergodic capacity is an averaged value over different orientations of BS and MS. We assume unit antenna gain at both BS and MS. We consider an $(8,8)$ cross (slant polarized with angle $45^{\circ}$ ) polarized MIMO system with $18 \mathrm{~dB}$ SNR. The inter-element spacing of the antennas at both ends is one wavelength.

Results in Fig. 5 are the ergodic capacity of the system vs the $g$ factor when changing the 2D AoA azimuth distributions but keeping the 3D AoA distributions as per the base line. It shows that distributions with the same AS perform very similarly. Two AS values of $35^{\circ}$ and $2^{\circ}$ are considered. For the $2^{\circ}$ case, results for all three distributions (Laplacian, von Mises and Gaussian) are virtually identical and at $35^{\circ}$ the differences are only slight. The ergodic capacity for all distributions overlap when $g$ is greater than $0 d B$. We also observe that ergodic capacity is very sensitive to the $\mathrm{g}$ parameter, especially for the low AS cases considered. The low AS case may arise when the MS receives signals from a narrow range due to building construction (i.e., the presence of windows). The ergodic capacity of the pure 3D propagation environment $(g=\infty)$ almost doubles the corresponding value for the 2D case $(g=0)$. For the large angle spread case, the 3D component still leads to the ergodic capacity increase of about 30 per cent. These results are intuitive, as the $3 \mathrm{D}$ distribution has a wider

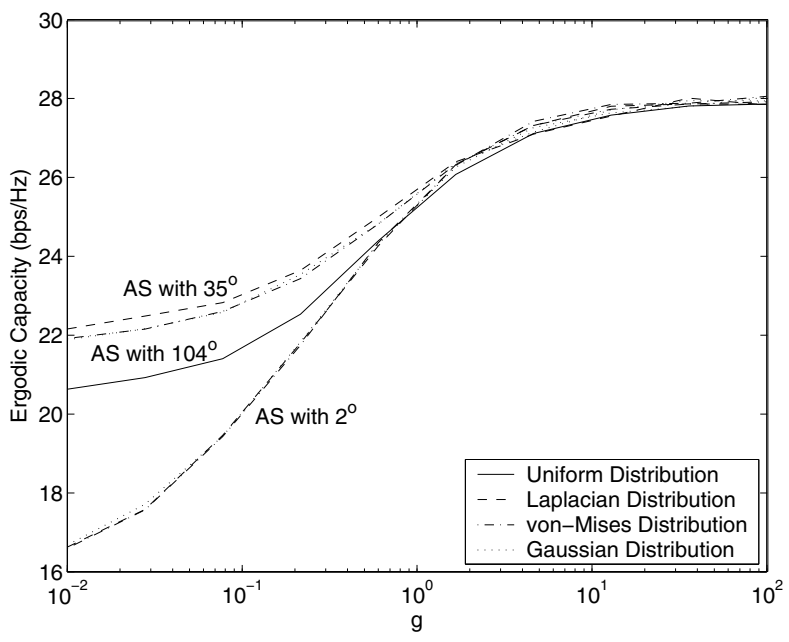

Fig. 5. Changing AoA distributions for the 2D component

angular spread, and thus leads to a better decorrelation of the signals at the different antenna elements.

Results in Fig. 6 show the ergodic capacity vs the AS of the 2D AoA distributions while keeping the 3D AoA distributions as per the base line. We observe that for every case the ergodic capacity reaches a peak at about $25^{\circ}$ AS. Above $25^{\circ}$ the ergodic capacity decreases with different slopes related to the type of distribution. For systems with narrow AS, the ergodic capacity is insensitive to the distribution, but for larger AS values the effect of the distribution is more pronounced. Variations in the ergodic capacity are larger for the uniform case. These results are largely driven by the correlation structure of the channel (not shown here for reasons of space) which shows an opposite pattern to Fig. 6. Hence, correlation decreases to around $25^{\circ}$ and then increases slightly. Such oscillatory correlation behaviour is well-known in time and space [1].

Results in Fig. 7 show the ergodic capacity of the system vs the $g$ factor when changing the 3D AoA elevation 


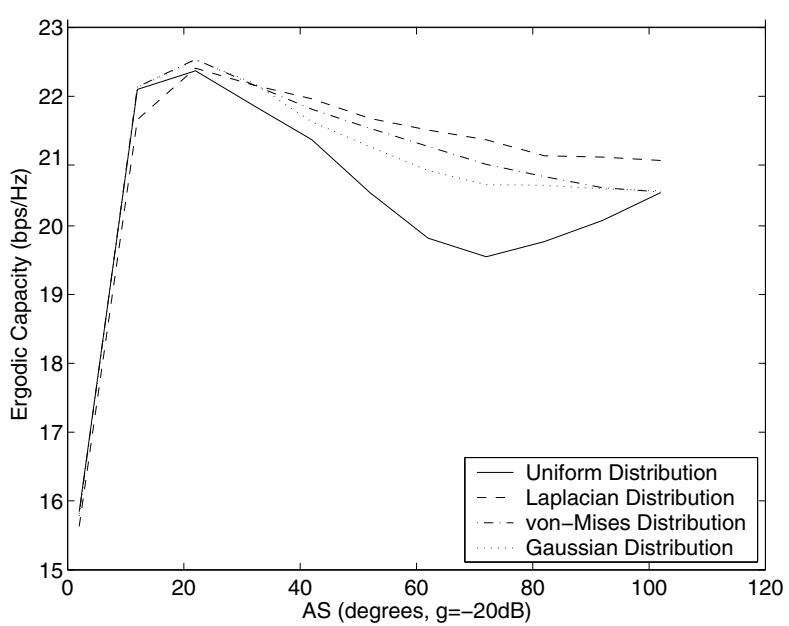

Fig. 6. Changing AS for the $2 \mathrm{D}$ component

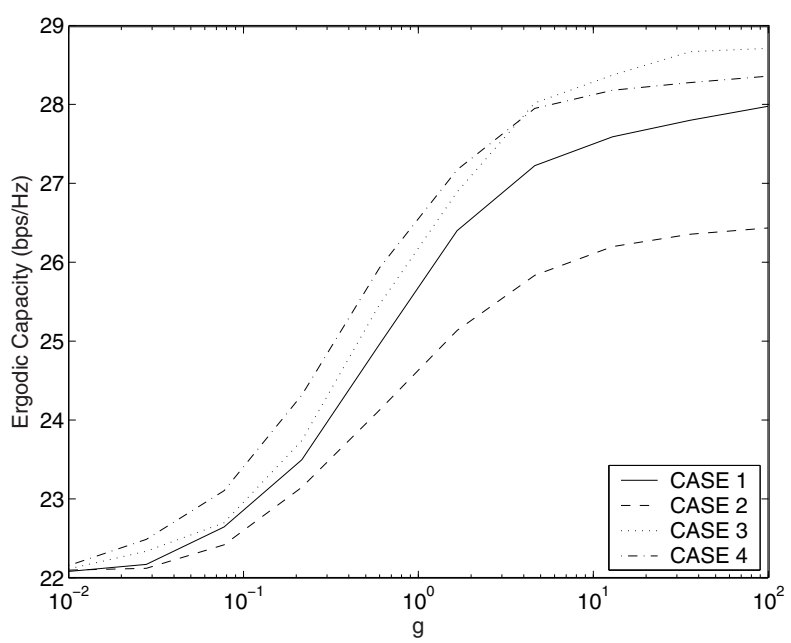

Fig. 7. Changing AoA distributions for the $3 \mathrm{D}$ component

distributions and keeping the 2D AoA distributions as per the base line. All cases start at the same point as they all have the same 2D distribution. The largest capacity increase vs $g$ is for the case when the incoming powers are concentrated on the top and bottom of the scattering sphere, followed by the uniform, spherical uniform and the case when power concentrates in the horizontal plane of the scattering sphere respectively. All these results show that the largest impact of the $3 \mathrm{D}$ component is for cases when the incoming power is coming from the top (or bottom) of the scattering sphere. Furthermore, ignoring the 3D component underestimates the ergodic capacity very significantly.

\section{CONCLUSIONS}

In this paper we present a novel composite channel model for a MIMO cross polarized channel. This consists of defining a composite channel coefficient that is a scaled sum of $2 \mathrm{D}$ and $3 \mathrm{D}$ components. The $3 \mathrm{D}$ component captures the environments when the elevation angle power spectrum is significant. The $2 \mathrm{D}$ and $3 \mathrm{D}$ components are scaled via a $g$ parameter to form the composite channel.

We show that ergodic capacity is very sensitive to the value of the $g$ parameter especially when the MS is in an environment with low AS. This is feasible as most of the energy reaching the MS may be coming from a certain range of angles due to building construction (e.g. the presence of windows) rather than uniformly from all directions. The consideration of elevation angle distributions is also important since differences in the concentration of elevation power can lead to significant changes in capacity. Similarly, different 2D azimuth AoA distributions can lead to different capacity values, especially for large AS values. Finally, omission of a significant 3D component in the channel can result in a significant underestimation of the ergodic capacity.

We believe these results will provide motivation to measure elevation angle distributions and eventually will be of value in practical layouts of MIMO systems.

\section{REFERENCES}

[1] M. Shafi, M. Zhang, A.L. Moustakis, P.J. Smith, A.F. Molisch, F. Tufvesson and S.H. Simon, "Polarized MIMO channels in 3D: Models, measurements and mutual information," IEEE $J$. Sel. Areas Commun., Mar. 2006, to appear.

[2] S. Kozono, T. Suruhara and M. Sakamoto, "Base station polarization diversity reception for mobile radio," IEEE Trans. Veh. Technol., vol. 33, no. 4, pp. 301-306, Nov. 1984.

[3] R.G. Vaughan, "Polarisation diversity in mobile communications," IEEE Trans. Veh. Technol., vol. 39, no. 3, pp. 177-185, June 1990.

[4] "Spatial channel model for multiple input multiple output MIMO simulations," 3GPP, vol. TR 25.996, v6.1.0, Sep. 2003.

[5] K. Kalliola, K. Sulonen, H. Latinen, O. Kivekas, J. Krogerus and P. Vainikainen, "Angular power distribution and mean effective gain of mobile antenna in different propagation environments," IEEE Trans. Veh. Technol., vol. 51, no. 5, pp. 823838, Sep. 2002.

[6] T. Taga, "Analysis for the mean effective gain of mobile antennas in land mobile radio environments," IEEE Trans. Veh. Technol., vol. 39, no. 2, pp. 117-131, May 1990.

[7] S. Qu and T. Yeap, "A three dimensional scattering model for fading channels in land mobile environment," IEEE Trans. Veh. Technol., vol. 48, no. 5, pp. 765-781, May 1999.

[8] C. A. Balanis, Antenna Theory: Analysis and Design. USA: John Wiley and Sons, 1982.

[9] C. F. T. Zwick and W. Wiesbeck, "A stochastic multipath channel model including path directions for indoor environments," IEEE J. Sel. Areas Commun., vol. 20, no. 6, pp. 1178-1192, Aug. 2002.

[10] M.B. Knudsen and G.F. Pedersen, "Spherical outdoor to indoor power spectrum model at the mobile terminal," IEEE J. Sel. Areas Commun., vol. 20, no. 6, pp. 1156-1169, Aug. 2002.

[11] I.E. Telatar, "Capacity of multi-antenna Gaussian channels," Europ. Trans. Telecommun., vol. 10, no. 6, pp. 585-595, 1999. 